\title{
Composição corporal de idosos segundo a antropometria
}

\author{
Body composition of elderly by anthropometry
}

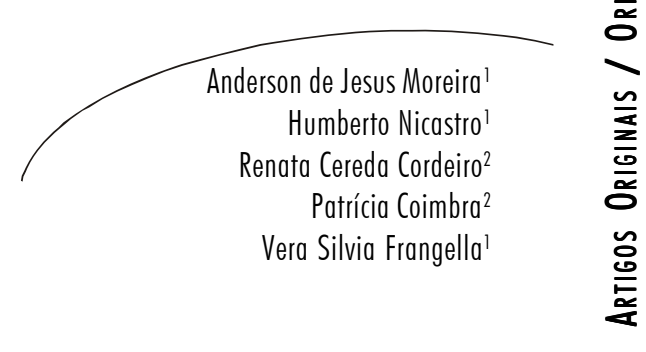

\section{Resumo}

Objetivo: Descrever o perfil antropométrico e a composição corporal de idosos por meio da antropometria, analisando a aplicabilidade das equações de predição de Petroski (1995) e de Durnin \& Womersley (1974). Métodos: Foram avaliados 37 idosos de ambos os gêneros. As medições antropométricas foram realizadas segundo as diretrizes da International Society for the Advancement of Kineanthropometry, e determinaram as variáveis massa corporal, estatura, oito dobras cutâneas e sete perímetros musculares. Calcularam-se índice de massa corporal (IMC), relação cintura-quadril (RCQ), somatório de cinco dobras cutâneas e densidade corporal pelas equações de predição para idosos de Petroski (1995) e de Durnin \& Womersley (1974) com conversão ao percentual de gordura (\%G) pela equação de Siri (1961). Para posterior discussão, aplicou-se o teste $\mathrm{t}$ Student $(\mathrm{p}<0,05)$. Resultados: As variáveis idade, massa corporal, estatura e IMC não diferiram entre os gêneros. $\mathrm{O}$ somatório de cinco dobras cutâneas resultou em eutrofia para ambos os gêneros. A RCQ indicou "risco moderado" para homens e "risco alto" para mulheres, de complicações metabólicas. A equação de Durnin \& Womersley (1974) subestimou nos indivíduos do gênero masculino e superestimou no gênero feminino o \%G obtido pela equação de Petroski (1995), respectivamente. Por ambas as equações, os indivíduos do gênero feminino apresentaram \%G superior aos do gênero masculino. Conclusões: Os indivíduos de ambos os gêneros apresentam elevada adiposidade corporal, especialmente na região

\author{
Palavras-chave: \\ Idoso. Perfil de \\ Saúde. \\ Antropometria. \\ Composição \\ Corporal. Estado \\ Nutricional.
}


omental. A equação de Petroski (1995) atendeu de modo satisfatório aos critérios de aplicação utilizados e mostrou ser adequada para a população em questão.

\section{Abstract}

Objective: To describe anthropometric profile and body composition of elderly by anthropometry and evaluate the applicability of Petroski (1995) and Durnin \& Womerseley (1974) protocols. Methods: We evaluated 37 elderly individuals of both genders. The anthropometric measurements were performed according to the guidelines of the International Society for the Advancement of Kineanthropometry and established the variables weight, height, eight skinfolds and seven muscle girths. It was calculated body mass index (BMI), waist-to-hip ratio (WHR), sum of five skinfolds and body density by Petroski (1995) and Durnin \& Womersley (1974) elderly protocols with the conversion to percentage of body fat $(\% \mathrm{BF})$ by Siri (1961) protocol. For further discussion, it was applied the Student $t$ test $(\mathrm{p}<0.05)$. Results: Age, weight, height and BMI did not differ between genders. The sum of five skinfolds resulted in normal range for both genders. The WHR indicated "moderate risk" for men, and "high risk" for women to develop metabolic complications. The Durnin \& Womersley (1974) protocol underestimated in male subjects and overestimated in female subjects the \%BF obtained by the equation of Petroski (1995), respectively. For both protocols, female subjects showed higher \%BF than male subjects. Conclusions: We conclude that individuals of both genders have high body fat, especially in the abdominal region. The Petroski (1995) protocol answered satisfactorily to the applying criteria used and showed to be adequate for this population.
Key words: Aged. Health Profile. Anthropometry. Body Composition. Nutritional Status.

\section{INTRODUÇÃO}

O fenômeno do envelhecimento populacional é observado em países desenvolvidos e em desenvolvimento, como resultado de conquistas nas áreas médicas e sociais, e conquistas tecnológicas. ${ }^{1}$ No Brasil, estimase uma população idosa de 30 milhões de pessoas em $2025 .^{2}$

Em diversos países, dentre eles o Brasil, o aumento no número de idosos observado nas últimas décadas tem gerado grande interesse em relação às alterações fisiológicas que afetam a conformação física e o declínio da capacidade funcional de alguns órgãos e sistemas à medida que a idade avança ${ }^{3-5}$ pois se sabe que o "envelhecimento saudável" melhora não somente a qualidade de vida, como também os custos direcionados aos cuidados à saúde. ${ }^{6}$ No Brasil, o envelhecimento é um fenômeno relativamente recente, contudo irreversível diante do comportamento declinante $\mathrm{da}$ fecundidade e $\mathrm{da}$ mortalidade registrado nas últimas décadas, que afeta diretamente o estado nutricional do indivíduo por todas as alterações que ocorrem no organismo, tais como diminui- 
ção dos botões gustativos, redução do olfato e da visão, diminuição da secreção salivar e gástrica, falha na mastigação (pela ausência de dentes ou próteses impróprias), constipação intestinal devido à redução da motilidade gastrintestinal.,

No tocante à ciência da antropometria, o processo de envelhecimento acarreta alterações nos compartimentos corporais, como a diminuição da massa corporal e da estatura, redução da massa livre de gordura (MLG) e modificação nos compartimentos de gordura corporal, onde o tecido adiposo periférico tende a diminuir e o central, ou omental, a aumentar. ${ }^{8,9}$ Sabe-se ainda que a avaliação antropométrica periódica pode refletir indiretamente na qualidade de vida da população idosa, uma vez que a força, como resultado do conteúdo muscular periférico, é um indicador de mortalidade em indivíduos saudáveis. ${ }^{10}$ Neste contexto, a antropometria tem-se mostrado importante método de avaliação do estado nutricional, pois, além de fornecer informações das medidas que podem refletir o estado de saúde e da qualidade de vida, é um método não-invasivo, de fácil e rápida execução e de baixo custo que estima os compartimentos corporais com razoável exatidão desde que realizada por um avaliador experiente. ${ }^{9}$

O presente estudo tem por objetivo traçar o perfil antropométrico e descrever a composição corporal de idosos por meio $\mathrm{da}$ antropometria, visto que dados antropométricos de referência em âmbito nacional de indivíduos com faixa etária superior a 60 anos são escassos na literatura. Secun- dariamente, o presente trabalho objetiva analisar a aplicabilidade das equações de predição de Petroski ${ }^{11}$ e Durnin \& Womersley, ${ }^{12}$ visto que a primeira é uma equação desenvolvida e validada para a população brasileira e a segunda, apesar de não ter sido desenvolvida primariamente para a população brasileira, é amplamente utilizada por profissionais no Brasil. ${ }^{13}$

\section{METODOLOGIA}

\section{Amostra}

O presente estudo caracteriza-se por ser transversal com coleta de dados primários. Foram avaliados 37 idosos com idade igual ou superior a 60 anos, sendo 27 do gênero feminino e 10 do gênero masculino. Como critérios de exclusão, foram considerados aqueles que estivessem edemaciados e/ou sem condições físicas para avaliação nutricional.

O convite aos indivíduos foi feito verbalmente e, após esclarecimentos sobre a metodologia, risco e objetivos do trabalho, os mesmos ou seus responsáveis assinaram um termo de consentimento livre e esclarecido. O projeto do estudo foi aprovado pelo Comitê de Ética em Pesquisa do Centro Universitário São Camilo com o protocolo número 003/06 e cumpriu os princípios éticos contidos na Resolução CONEP no ${ }^{\circ}$ 196/96. ${ }^{14}$

\section{Avaliação antropométrica}

As medições antropométricas foram realizadas segundo as diretrizes da International 
Society for the Advancement of Kineanthropometry (IS AK). ${ }^{15}$ Foram determinadas as seguintes variáveis antropométricas: massa corporal, estatura, oito dobras cutâneas (biceptal, triceptal, subescapular, supra-ilíaca, supra-espinal, abdominal, coxa medial e panturrilha) e sete perímetros musculares (tórax, braço relaxado, cintura, abdominal, quadril, coxa medial e panturrilha). As medidas foram triplicadas por um avaliador experiente e utilizou-se como resultado a média aritmética das mesmas.

A massa corporal foi obtida por meio de uma balança digital da marca Filizola ${ }^{\circledR}$, com capacidade de $150 \mathrm{~kg}$ e precisão de $100 \mathrm{~g}$; a estatura, por meio de um estadiômetro vertical fixo à balança; a espessura das dobras cutâneas foi aferida, em triplicata, do lado direito do indivíduo, utilizando-se um

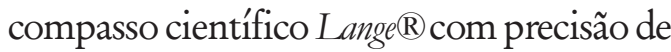
$1 \mathrm{~mm}$ e os perímetros musculares com o auxilio de uma fita antropométrica Gulick ${ }^{\circledR}$ com precisão de $1 \mathrm{~mm}$.

O índice de massa corporal (IMC) foi calculado considerando-se a razão entre a massa corporal (em kg) e a estatura (em metros) ao quadrado $\left(\mathrm{kg} / \mathrm{m}^{2}\right)$ e classificado segundo os pontos de corte propostos pela NSI - Nutrition Screening Initiative. ${ }^{16} \mathrm{O}$ perímetro da cintura foi classificado segundo a WHO - World Health Organization. ${ }^{17} \mathrm{~A}$ relação entre o perímetro da cintura e do quadril foi classificada segundo Lohman. ${ }^{18}$

Para a determinação da composição corporal, foi aplicada a equação de predição de densidade corporal (DC) proposta por Petroski, ${ }^{11}$ com utilização de quatro dobras cutâneas para o gênero feminino $(D C=$ 1,02902361-0,00067159* (Dobra cutânea subescapular + Dobra cutânea triceptal + Dobracutânea supra-ilíaca + Dobra cutânea da panturrilha) $+0,00000242 \times$ (Dobra cutânea subescapular + Dobra cutânea triceptal + Dobra cutânea supra-iliaca + Dobra cutânea da panturrilha) ${ }^{2}$ 0,0002073* (Idade) $-0,00056009 *$ (Massa corporal) $+0,00054649 *$ (Estatura)); e para o gênero masculino $(D C=1,10726863$. 0,00081201 × (Dobra cutânea subescapular + Dobra cutânea triceptal + Dobra cutânea suprailíaca + Dobra cutânea da panturrilha) + 0,00000212 $\times$ (Dobra cutânea subescapular + Dobra cutânea triceptal + Dobra cutânea suprailíaca + Dobra cutânea da panturrilha $)^{2}$. 0,00041761* (Idade)). Em caráter comparativo, aplicou-se também a equação de DC proposta por Durnin \& Womersley, ${ }^{12}$ que utiliza quatro dobras cutâneas para o gênero feminino $(D C=1,1339-0,0648 x$ $\log _{10}$ (Dobra cutanea subescapular + Dobra cutânea triceptal + Dobra cutânea supra-ilíaca + Dobra cutânea biceptal)) e para o gênero masculino: (DC $=1,1765-0,0744 \times \log _{10}$ (Dobra cutânea subescapular + Dobra cutânea triceptal + Dobra cutânea supra-ilíaca + Dobra cutânea biceptal)). Posteriormente, para conversão da DC em percentual de gordura corporal $(\% \mathrm{G})$, foi utilizada a equação de Siri: ${ }^{19}(\% G=[4,95$ ( DC) $-4,50] \times 100)$ ).

Análise estatística

A análise dos dados foi conduzida pelo Software Statistica versão 7.0 e realizada de 
modo descritivo para todas as variáveis em cada indivíduo do grupo de estudo. Os dados são avaliados pela análise de tendência central (média), variabilidade (desvio padrão) e coeficiente de variação. Para posterior discussão, aos resultados obtidos aplicou-se o teste $t$ Student com nível de significância adotado de $p<0,05$.

\section{RESULTADOS}

Na tabela 1, são apresentadas as características da amostra por meio dos valores médios e respectivos desvios padrão das variáveis idade, massa corporal, estatura e IMC dos indivíduos, segundo o gênero. Não houve diferenças estatísticas intergrupos.

Tabela 1- Valores médios, desvios-padrão e coeficiente de variação das características gerais da amostra segundo o gênero (idade, massa corporal, estatura e IMC). São Paulo, 2007.

\begin{tabular}{lcccc}
\hline \multirow{2}{*}{ Variável } & \multicolumn{2}{c}{ Masculino $(\mathrm{n}=10)$} & \multicolumn{2}{c}{ Feminino $(\mathrm{n}=27)$} \\
\cline { 2 - 5 } & Média \pm DP & CV $(\%)$ & Média \pm DP & CV $(\%)$ \\
\hline Idade (anos) & $74,6 \pm 7,8$ & 10,4 & $69,0 \pm 6,6$ & 9,6 \\
Massa corporal $(\mathrm{kg})$ & $73,4 \pm 12,1$ & 16,5 & $67,9 \pm 11,7$ & 17,2 \\
Estatura $(\mathrm{cm})$ & $166 \pm 0,04$ & 16,8 & $152 \pm 0,05$ & 3,4 \\
IMC $\left(\mathrm{kg} / \mathrm{m}^{2}\right)$ & $26,48 \pm 4,04$ & 27,0 & $27,28 \pm 4,93$ & 16,8 \\
\hline
\end{tabular}

Análise descritiva. Dados apresentados em média \pm desvio-padrão. $\mathrm{DP}=$ desvio padrão; $\mathrm{CV}=$ coeficiente de variação; IMC $=$ índice de massa corporal. Sem diferenças estatísticas intergrupos segundo o teste $t$ Student.

A média, o desvio padrão e o coeficiente de variação das variáveis dobras cutâneas e perímetros musculares e do somatório de dobras cutâneas para avaliação da gordura corporal total ( $\Sigma 5 \mathrm{DC})$ coletados neste estudo, segundo o gênero, são refe- ridos na tabela 2. De modo geral, nota-se que, em todos os indivíduos, independentemente do gênero, as variáveis médias de dobras cutâneas apresentam CV superior em relação ao observado nas médias dos perímetros musculares. 
Tabela 2 - Média, desvio-padrão e coeficiente de variação das variáveis dobras cutâneas e perímetros musculares dos indivíduos participantes do estudo, segundo o gênero. São Paulo, 2007.

\begin{tabular}{lcccc}
\hline \multirow{2}{*}{ Variável } & \multicolumn{2}{c}{ Masculino $(\mathrm{n}=10)$} & \multicolumn{2}{c}{ Feminino $(\mathrm{n}=27)$} \\
\cline { 2 - 5 } & Média $\pm \mathrm{DP}$ & $\mathrm{CV}(\%)$ & Média $\pm \mathrm{DP}$ & $\mathrm{CV}(\%)$ \\
\hline Dobra cutânea triceptal $(\mathrm{mm})$ & $11,5 \pm 4,2$ & 36,7 & $21,9 \pm 5,9$ & 27,0 \\
Dobra cutânea biceptal $(\mathrm{mm})$ & $8,0 \pm 2,7$ & 33,9 & $13,1 \pm 4,5$ & 34,7 \\
Dobra cutânea subescapular $(\mathrm{mm})$ & $17,8 \pm 7,2$ & 40,3 & $21,3 \pm 6,4$ & 29,9 \\
Dobra cutânea suprailíaca $(\mathrm{mm})$ & $17,2 \pm 6,2$ & 36,1 & $24,7 \pm 7,8$ & 31,5 \\
Dobra cutânea supraespinal $(\mathrm{mm})$ & $14,4 \pm 5,0$ & 35,0 & $24,1 \pm 7,4$ & 30,4 \\
Dobra cutânea abdominal $(\mathrm{mm})$ & $21,4 \pm 7,2$ & 33,9 & $29,4 \pm 7,2$ & 24,4 \\
Dobra cutânea coxa medial $(\mathrm{mm})$ & $16,9 \pm 5,6$ & 32,9 & $24,8 \pm 7,3$ & 29,4 \\
Dobra cutânea panturrilha $(\mathrm{mm})$ & $12,9 \pm 3,6$ & 27,9 & $22,3 \pm 4,8$ & 21,5 \\
E5DC (mm)* & $84,8 \pm 26,8$ & 31,6 & $122,0 \pm 27,1$ & 22,2 \\
Perímetro braquial $(\mathrm{cm})$ & $30,4 \pm 3,7$ & 12,1 & $31,0 \pm 4,3$ & 13,8 \\
Perímetro da cintura $(\mathrm{cm})$ & $96,5 \pm 9,6$ & 10,0 & $95,2 \pm 11,1$ & 11,6 \\
Perímetro abdominal $(\mathrm{cm})$ & $94,6 \pm 7,7$ & 8,1 & $103,0 \pm 12,0$ & 11,6 \\
Perímetro do quadril $(\mathrm{cm})$ & $99,1 \pm 10,3$ & 10,4 & $102,9 \pm 9,2$ & 8,9 \\
Perímetro da coxa medial $(\mathrm{cm})$ & $42,5 \pm 4,9$ & 11,5 & $47,3 \pm 6,4$ & 13,6 \\
Perímetro da panturrilha $(\mathrm{cm})$ & $36,2 \pm 5,4$ & 14,9 & $37,8 \pm 4,1$ & 10,8 \\
Perímetro do torácico $(\mathrm{cm})$ & $99,3 \pm 7,9$ & 7,9 & $95,9 \pm 7,7$ & 8,0 \\
RCQ** & $0,97 \pm 0,06$ & 5,8 & $0,92 \pm 0,05$ & 5,9 \\
\hline
\end{tabular}

Análise descritiva. Dados apresentados em média + desvio-padrão. $\mathrm{DP}=$ desvio-padrão; $\mathrm{CV}=$ coeficiente de variação; *Somatório de 5 dobras cutâneas. **Relação cintura-quadril.

As figuras 1 e 2 descrevem os valores médios de \%G, massa gorda (MG) e massa livre de gordura (MLG), por diferentes equações, observados nos indivíduos do sexo masculino e feminino, respectivamente. Observa-se que o \%G dos indivíduos do gênero masculino calculado segundo Petroski ${ }^{11}$ foi significativamente superior em relação ao valor obtido pela equação de Durnin \& Womersley. ${ }^{12} \mathrm{O}$ oposto foi observado nos indivíduos do gênero feminino, onde o $\% G$ calculado por Petroski ${ }^{11}$ foi significativamente menor em relação ao calculado por Durnin \& Womersley. ${ }^{12}$ Consequentemente, todas as diferenças estatísticas referentes ao \%G, com exceção dos indivíduos do gênero masculino, refletiram em valores significativamente diferentes para a MG. Adicionalmente, na figura 3 foi possível observar que os indivíduos do gênero feminino apresentaram $\% G$ significativamente superior em relação ao gênero masculino, quando comparados os valores obtidos pela equação de Petroski. ${ }^{11}$ 


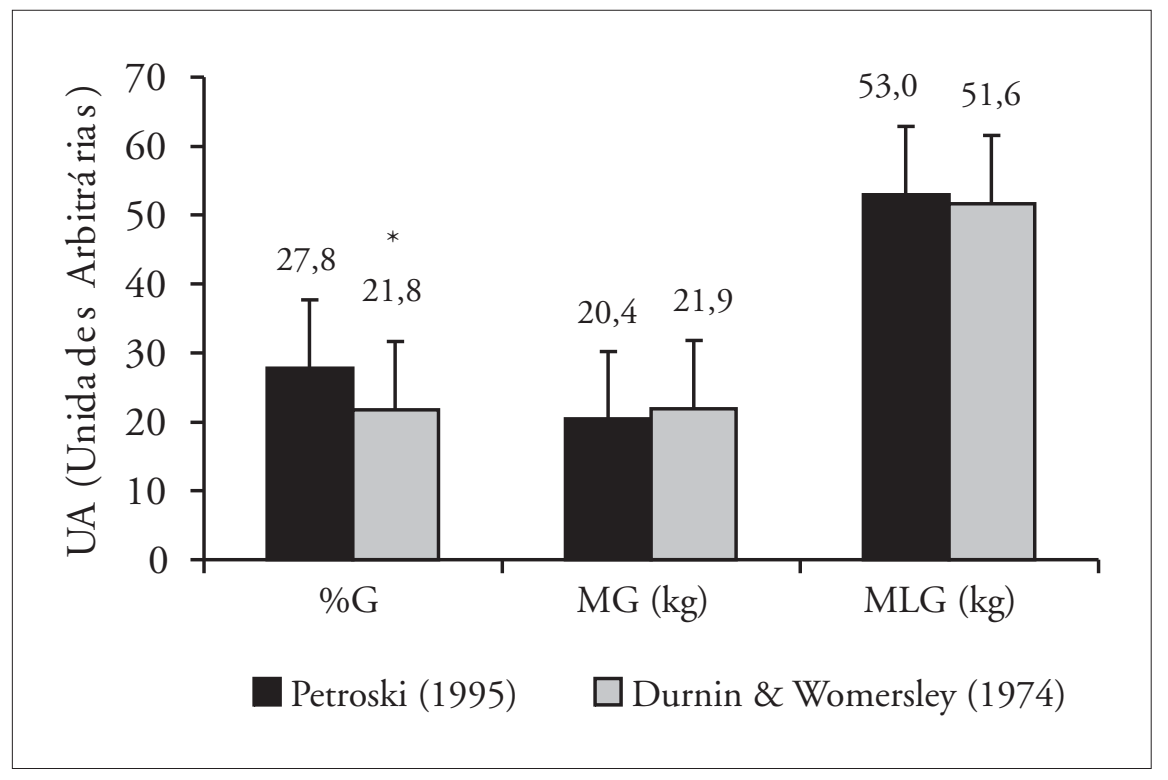

Figura 1 - Valores médios do percentual de gordura (\%G), massa gorda (MG) e massa livre de gordura (MLG) dos indivíduos do gênero masculino $(n=10)$ por diferentes equações de predição. São Paulo, 2007. *p <0,05 entre as equações.

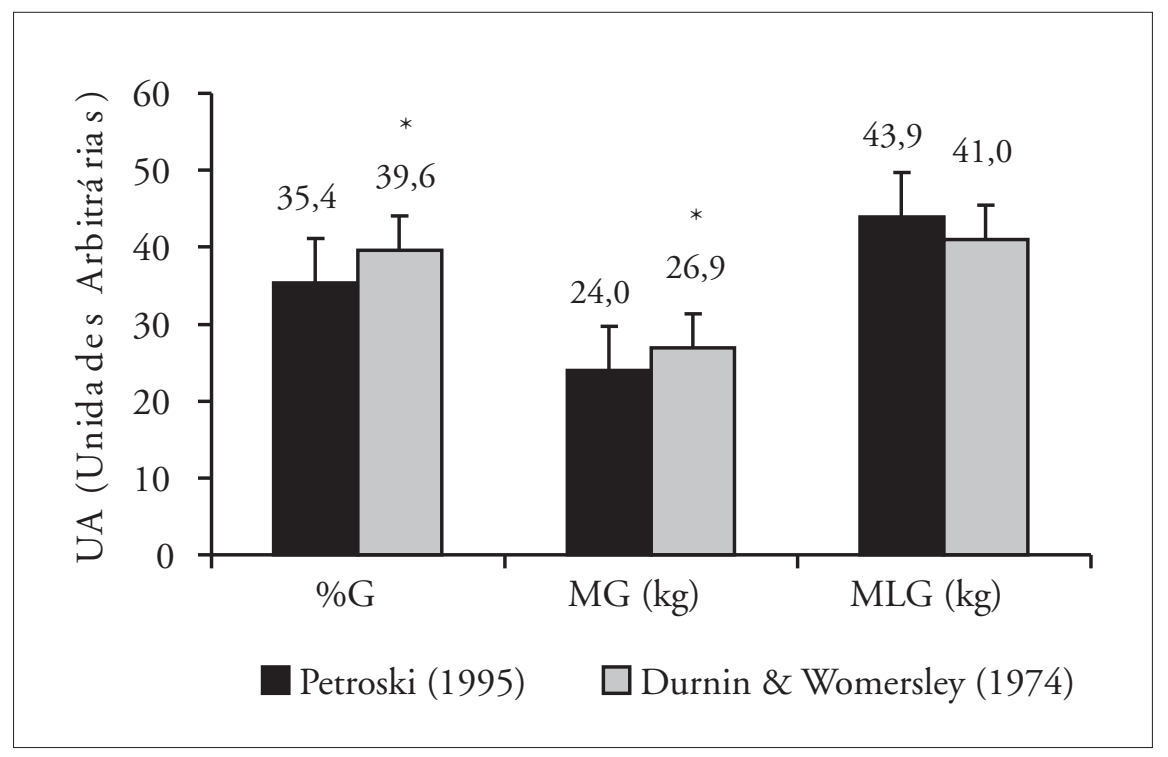

Figura 2 - Valores médios do percentual de gordura (\%G), massa gorda (MG) e massa livre de gordura (MLG) dos indivíduos do gênero feminino $(n=27)$ por diferentes equações de predição. São Paulo, 2007. *p <0,05 entre as equações. 


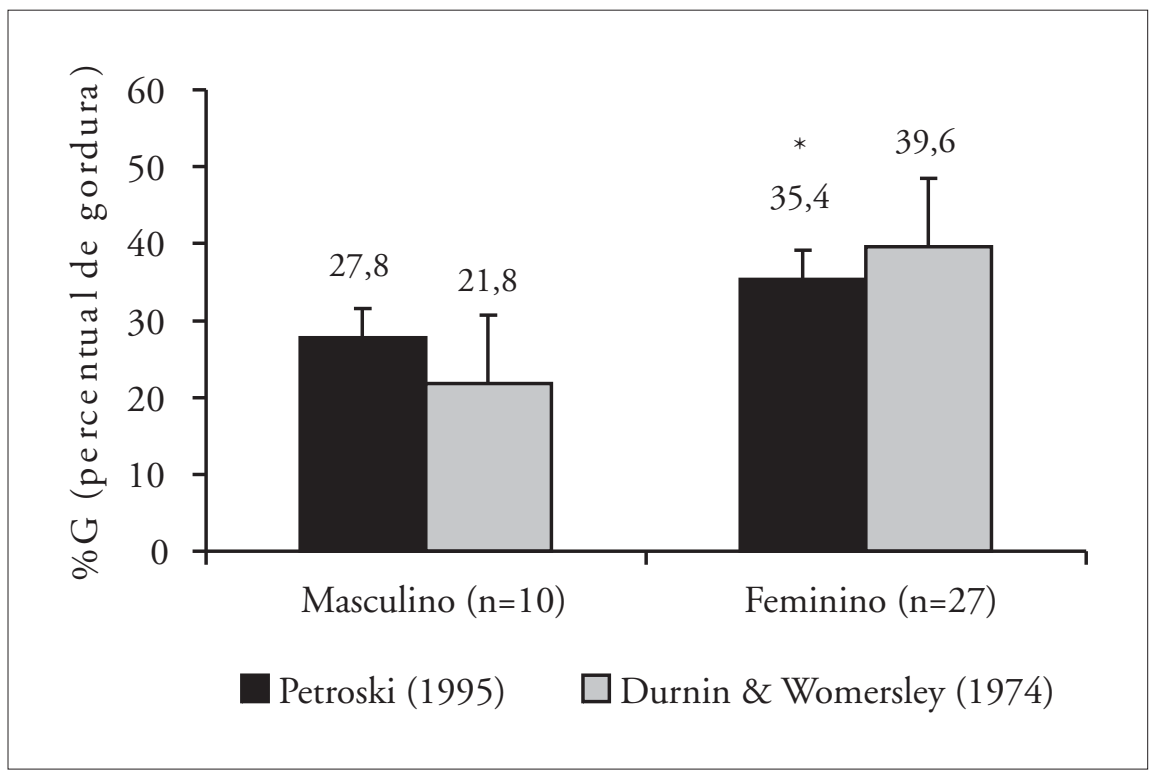

Figura 3 - Valores médios do percentual de gordura $(\% \mathrm{G})$ dos indivíduos segundo o gênero por diferentes equações de predição. São Paulo, 2007. *p $<0,05$ entre os gêneros pela mesma equação de predição.

\section{DISCUSSÃO}

Como dito anteriormente, à época dessa pesquisa eram escassos os estudos realizados em âmbito nacional que traçavam o perfil antropométrico e estimam a composição corporal de idosos por meio da antropometria com equações previamente validadas. Assim, pode-se considerar este trabalho como pioneiro na linha de pesquisa em questão, sobretudo no tocante ao registro de variáveis antropométricas e predição da composição corporal por equação específica.

Do total de idosos avaliados, $73 \%$ pertenciam ao gênero feminino, o que demonstra que nossos dados condizem com o nú- mero absoluto de mulheres idosas no Brasil quando confrontado com o de homens de 65 anos ou mais. Essa situação decorre da existência de mortalidade diferencial por sexo que prevalece há muito tempo na população brasileira. Desde 1950, as mulheres possuem maior esperança de vida. Em 1980, enquanto a expectativa de vida para os homens era de 59 anos, para as mulheres era de 65 anos. Já em 1991, essa diferença cresceu para sete anos. ${ }^{20}$

De modo geral, podemos classificar a população deste estudo como eutrófica segundo a classificação proposta pelo $\mathrm{NSI}^{16}$ (baixo peso $=<22$; eutrofia $=22 \mathrm{a}<27$; sobrepeso $=27 \mathrm{a}<30$; obesidade $=>30$ ), 
considerando que os indivíduos do gênero feminino estão na faixa limítrofe da eutrofia. Tratando-se de estudos nacionais, nossos resultados de IMC são corroborados pelos estudos de Santos \& Sichieri, ${ }^{21}$ nos quais o valor médio de IMC para mulheres de 70 a 79,9 anos foi de $24,5 \mathrm{~kg} / \mathrm{m}^{2}$ e para homens de 60 a 69,9 anos de $25,2 \mathrm{~kg} / \mathrm{m}^{2}$, e de Menezes \& Marucci ${ }^{9}$ que encontraram IMC médio de $23,7 \mathrm{~kg} / \mathrm{m}^{2}$ para mulheres de 70 a 79 anos. Elsangedy et al..$^{8}$ encontraram um valor médio superior em mulheres idosas de IMC de $29,92 \mathrm{~kg} / \mathrm{m}^{2}$. Contudo, a divergência deste último em relação ao nosso estudo se deve, em parte, ao fato de que a população estudada não era saudável, pois apresentava o quadro de hipertensão arterial sistêmica. Já no estudo de caráter internacional de Arroyo et al., ${ }^{22}$ os valores de IMC encontrados para homens e mulheres idosas condizem com os apresentados em nosso estudo.

Apesar de nossos resultados serem um tanto quanto similares a estudos nacionais e internacionais, tal dado deve ser interpretado cuidadosamente, visto que as alterações nos compartimentos corporais em decorrência da idade podem estabelecer um diferente diagnóstico, o que interferiria no planejamento de uma intervenção nutricional e/ou física.

Como já descrito anteriormente, os métodos duplamente indiretos de avaliação possuem algumas vantagens que os fazem amplamente utilizados. Assim, a tabela 2 tem o importante papel de registrar e estabelecer medidas antropométricas de referên- cia com razoável exatidão. No contexto internacional, apenas Baumgartner et al. ${ }^{23}$ realizaram o registro de variáveis antropométricas por meio da antropometria em 98 idosos de ambos os gêneros com idade entre 65 e 94 anos e, dentre as variáveis coletadas, as dobras cutâneas triceptal e abdominal se assemelham aos valores obtidos em nosso estudo. Apesar de as demais medidas diferirem em relação às apresentadas em nosso trabalho, dois pontos similares interessantes foram encontrados: uma maior variação no valor de dobras cutâneas e menor variação de perímetros musculares dentro da amostra.

Recentemente, Nicastro et al. ${ }^{24}$ e Borba et al..$^{25}$ descreveram uma interessante correlação entre diferentes variáveis antropométricas localizadas sobre o mesmo ponto anatômico para compreender a variação tecidual em alguns compartimentos corporais. Partindo deste princípio, observa-se nessa população variação tecidual acentuada, uma vez que o CV para as dobras cutâneas encontra-se alto, ao contrário do apresentado para os perímetros musculares. Mais especificamente, é possível observar que na região braquial a população estudada, em ambos os gêneros, não se apresenta homogeneidade, uma vez que o perímetro braquial apresenta baixo e a dobra cutânea alto $\mathrm{CV}$, indicando assim uma possível variação tecidual.

A mesma análise pode ser feita para a região abdominal, importante para a prevenção e diagnóstico de comorbidades crônicas como diabetes tipo 2, hipertensão arterial e distúrbios do metabolismo lipídico. ${ }^{26,27}$ 
Para o perímetro abdominal, nota-se que esta variável apresenta baixo CV e que indivíduos dos gêneros masculino e feminino encontram-se em risco alto para o desenvolvimento de complicações cardiovasculares segundo a $\mathrm{WHO},{ }^{17}$ respectivamente. Ao correlacionar tal variável com a dobra cutânea triceptal, também é possível observar que há uma possível variação tecidual, pois esta última apresenta alto CV. Contudo, apesar de haver esta possível variação, os indivíduos encontram-se de modo geral em risco cardiovascular. Uma das hipóteses para o aumento do perímetro da cintura, principalmente em mulheres, refere-se a algumas alterações comportamentais que são características nesta população, como a redução nos níveis de atividade física e uma alimentação desequilibrada, as quais contribuem também para o comprometimento do perfil antropométrico. ${ }^{28}$

Confirmando o diagnóstico estabelecido pelo perímetro abdominal, o valor médio obtido pela RCQ em homens apresentou classificação de "risco moderado" e em mulheres "risco alto" para o desenvolvimento de complicações metabólicas, respectivamente, segundo Lohman. ${ }^{18}$ Nossos resultados são confirmados por estudos nacionais ${ }^{21} \mathrm{e}$ internacionais ${ }^{23}$ que utilizaram esta variável para a avaliação.

Segundo Costa, ${ }^{29}$ o somatório de dobras cutâneas é considerado um excelente indicador para estimar a gordura subcutânea, por ser previamente validado para a população brasileira. Na população de nosso estudo, os indivíduos de ambos os gêneros apresentaram classificação "normal” para a somatória, de acordo com Costa. ${ }^{29}$ Tal diagnóstico pode ser explicado pelo fato de as dobras cutâneas apresentarem um alto CV em ambos os gêneros, o que consequentemente se reflete também num alto $C V$ para a somatória de dobras. Portanto, o resultado em questão deve ser interpretado cuidadosamente e de maneira não isolada. Além disso, este é o primeiro estudo que registra o percentual de gordura corporal total por somatório de dobras cutâneas em idosos.

As equações de predição são frequentemente utilizadas na avaliação de indivíduos ou grupos populacionais, sem a realização de validação prévia na amostra que se propõe avaliar. Esse procedimento pode implicar erros sistemáticos e levar à obtenção de estimativas menos confiáveis. Portanto, verificar a validade das equações para a população que se pretende avaliar é de extrema importância para a obtenção de estimativas mais confiáveis. ${ }^{13}$ Assim como o somatório de dobras cutâneas, a equação de Petroski ${ }^{11}$ apresenta validade para a população brasileira. Quando analisamos as equações para cálculo do \%G, verificamos que há diferença significativa entre as mesmas.

$\mathrm{Na}$ equação proposta por Petroskii, ${ }^{11}$ há diferença estatística significativa entre homens e mulheres, o que é previsto, pois se sabe que indivíduos do gênero feminino possuem diferenças em termos de distribuição dos compartimentos corporais com maior adiposidade glúteo-femoral e menor massa muscular esquelética. ${ }^{30}$ Entretanto, o oposto ocorre na equação proposta por 
Durnin \& Womersley. ${ }^{12}$ Quando analisamos as duas equações para o mesmo gênero, verificou-se que Durnin \& Womersley ${ }^{12}$ superestimou e subestimou significativamente o $\% \mathrm{G}$ em mulheres e homens, respectivamente, em relação ao resultado apresentado por Petroski. ${ }^{11}$

Estudos nacionais que determinam o $\% \mathrm{G}$ total de idosos por meio do somatório de dobras cutâneas e por equações de predição são escassos. Neste último parâmetro, nossos resultados podem ser comparados a estudos internacionais que utilizaram métodos considerados "padrão-ouro" de avaliação da composição corporal. Baumgartner et al..$^{23}$ estimaram a composição corporal de idosos saudáveis por meio do modelo de quatro compartimentos corporais baseado na hidrodensitometria, diluição de ${ }^{3} \mathrm{H}_{2} \mathrm{O}$ e da fóton absorção dual e encontram valor médio de $\% \mathrm{G}$ de $23,32 \%$ em homens e $31,53 \%$ em mulheres, valores que se aproximam aos obtidos em nosso estudo. Arroyo et al. ${ }^{22}$ avaliaram a composição corporal de idosos por DEXA e encontraram $\% G$ médio para homens e mulheres com idade inferior a 75 anos de $24,9 \%$ e $27,8 \%$, respectivamente, dados que se aproximam ao de nosso estudo, sobretudo na população do gênero masculino.

\section{CONCLUSÕES}

A ampliação de estudos de composição corporal e perfil antropométrico em idosos utilizando as variáveis dobras cutâneas e perímetros musculares de modo mais abran- gentes favorecem o melhor diagnóstico do estado nutricional desta população, o que facilita o planejamento e a elaboração de uma conduta a ser aplicada.

Os resultados observados em nossa população de estudo sugerem atenção quanto à necessidade de se intervir nutricionalmente na rotina desta população, para que estratégias alimentares possam auxiliar na redução dos valores antropométricos elevados, colaborando assim para a diminuição de riscos de patologias associadas à obesidade. Os valores para classificação do IMC utilizados neste estudo são similares aos propostos pelo Ministério da Saúde,${ }^{31}$ demonstrando que os pontos de corte adotados atendem à população brasileira. Adicionalmente, a equação proposta por Petroski ${ }^{11}$ para a avaliação do $\% \mathrm{G}$, neste estudo, atendeu de modo satisfatório aos critérios de aplicação utilizados e mostrou ser adequada, com base nos estudos internacionais, para a população em relação à equação proposta por Durnin \& Womersley, ${ }^{12}$ indicando uma alternativa positiva e mais próxima da realidade brasileira. Contudo, há necessidade de realizar novos estudos que verifiquem a eficiência dessa e de outras metodologias, bem como uma discussão profunda entre a comunidade científica, antes que sejam adotadas em larga escala.

Vale ressaltar que a principal limitação do presente estudo é o baixo número amostral, o que impede que sejam realizadas análises estatísticas específicas com estratificação da amostra. Vale destacar, ainda, que a mensuração de medidas antropométricas em 
idosos, principalmente dobras cutâneas e perímetros, sofre interferência do processo de perda de tecido muscular esquelético.
Estudos futuros podem ampliar o número amostral e desenvolver mais análises acerca deste importante e interessante tema.

\section{REFERÊNCIAS}

1. Mendes MRSSB, et al. A situação social do idoso no Brasil: uma breve consideração. Acta paulista de enfermagem 2005; 8(4): 422-6.

2. Menezes TN, Lopes FJN, Marucci MFN. Estudo domiciliar da população idosa de Fortaleza/CE: aspectos metodológicos e características demográficas. Revista brasileira de epidemiologia 2007; 10(2): 168-77.

3. Fillenbaum GG. The wellbeing of the elderly: approaches to multidimensional assessment. Geneva: WHO; 1984.

4. Guralnik JM, Lacroix AZ. Assessing physical function in older populations. In: Wallace RB, Woolson RF. The epidemiologic study of the elderly. New York: Oxford University Press; 1992.

5. Jette AM, Branch L. Impairment and disability in the aged. J Chronic Dis 1985; 38(1): 59-65.

6. Campanella LCA, et al. Relação entre padrão alimentar e estado nutricional de idosos hospitalizados. Revista brasileira de nutrição clínica 2007; 22(2): 100-6.

7. Garcia ANM, RomaniSAM, Lira PIC. Indicadores antropométricos na avaliação nutricional de idosos: um estudo comparativo. Rev Nutr 2007; 20(4): 371-378.

8. Elsangedy HM et al. Avaliação do perfil antropométrico de idosas portadoras de hipertensão arterial. Arquivos de ciências da saúde da UNIPAR 2006; 10(2): 77-82.

9. Menezes TN, Marucci MFN. Antropometria de idosos residentes em instituições geriátricas, Fortaleza, CE. Rev Saúde Pública 2005; 39(2): 163-8.

10. Metter EJ, et al. Skeletal muscle strength as a predictor of all-cause mortality in healthy men.J Gerontol 2002; 57(10) B359-65.

11. Petroski EL. Desenvolvimento e validação de equações generalizadas para a estimativa da densidade corporal em adultos. 1995. [tese]. Santa Maria: Universidade Federal de Santa Maria; 1995.

12. Durnin JV, Womersley J. Body fat assessed from total body density and its estimation from skinfold thickness: measurements on 481 men and women aged from 16 to 72 years. Br J Nutr 1974; 32(1): 77-97.

13. Rezende FAC, et al. Aplicabilidade de equações na avaliação da composição corporal da população brasileira. Rev Nutr 2006; 19(3): 357-67.

14. Brasil. Ministério da Saúde. Conselho Nacional de Saúde. Comissão Nacional de Ética em Pesquisa. Resolução 196/96 sobre pesquisa envolvendo seres humanos. Brasília; 1996.

15. Marfell-Jones M, et al. International standards for anthropometric assessment. Potchefstroom: ISAK; 2006.

16. Nutrition Screening Initiative (NSI-2002). A Physician's Guide to Nutrition in Chronic Disease Management for Older Adults. Leawood (KS): American Academy of Family Physicians; 2002.

17. World Health Organization. Defining the problem of overweight and obesity. In: World Health Organization. Obesity: 
preventing and managing the global epidemic: report of a Who Consultation. Geneva; 2000. (WHO technical report series, 894).

18. Lohman, TG, Roche AF, Martorell R. Anthropometric standardization reference manual. Champaign: Human Kinetics Books; 1988.

19. Siri WE. Body composition from fluid space and density: analysis of method in: Brozek J, Henschel A. Techniques for measuring body composition. Washington: National Academy of Sciences; 1961.

20. Santos SR, et al. Qualidade de vida do idoso na comunidade: aplicação da Escala de Flanagan. Rev Lat Am Enfermagem 2002; 10(6): 757-64.

21. Santos DM, Sichieri R. Índice de massa corporal e indicadores antropométricos de adiposidade em idosos. Rev Saude Publica 2005; 39(2): 163-8.

22. Arroyo $\mathrm{P}$, et al. Indicadores antropométricos, composición corporal y limitaciones funcionales en ancianos. Rev Med Chil 2007; 135(7): 846-54.

23. Baumgartner RN, et al. Body composition in elderly people: effect of criterion estimates on predictive equations. Am J Clin Nutr 1991; 53(6): 1345-53
24. Nicastro $\mathrm{H}$, et al. Perfil antropométrico de indivíduos com lesão medular. Nutrire 2008; 33(1): 73-86.

25. Borba CT, Nicastro H, Viebig RF. Anthropometric characteristics, body composition and somatotype of Brazilian elite fencers. Gazz Med Ital 2009; 168: 316.

26. Kahn SE, Hull RL, Utzschneider KM. Mechanisms linking obesity to insulin resistance and type 2 diabetes. Nature 2006; 444(7): 840-6.

27. Scherer PE. Adipose tissue: from lipid storage compartment to endocrine organ. Diabetes 2006; 55(6): 1537-45.

28. Pena M, Bacallao J. Obesity among the poor: an emerging problem in Latin America and the Caribean. New York: Pan American Health Organization; 2000.

29. Costa RF. Composição corporal: teoria e prática da avaliação. São Paulo: Manole; 2001.

30. McArdle WD, Katch FI, Katch VL. Nutrição para o desporto e o exercício. Rio de Janeiro: Guanabara Koogan; 2001.

31. Brasil. Ministério da Saúde. CoordenaçãoGeral da Política de Alimentação e Nutrição; Organização Pan-Americana da Saúde; Fundação Oswaldo Cruz. Vigilância Alimentar e Nutricional-SISVAN: orientações básicas para a coleta, o processamento, a análise de dados e a informação em serviços de saúde. Brasília: Ministério da Saúde; 2004. Série A Normas e Manuais Técnicos. 


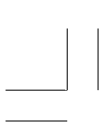

